\title{
TENSOR PRODUCTS OF LOCALLY CONVEX MODULES AND APPLICATIONS TO THE MULTIPLIER PROBLEM
}

\author{
BY \\ ROGER RIGELHOF $\left({ }^{1}\right)$
}

\begin{abstract}
In this paper we present a representation theorem for the tensor product of locally convex modules. This theorem has a number of consequences in the study of the multiplier problem in harmonic analysis, and the remainder of the paper is devoted to these applications.
\end{abstract}

1. Introduction. In a previous paper [9] concerned with induced representations of locally compact groups we studied tensor products of locally convex spaces which are modules over a topological algebra. In the introduction to that paper we mentioned that this study also provides an appropriate setting for the multiplier problem in Harmonic Analysis and this is the subject with which we now deal. This is the paper promised in the introduction of [9].

We begin by stating a classical multiplier problem and then we will consider more general situations. Let $\Gamma$ be a locally compact abelian group, $A(\hat{I})$ the algebra of functions on the character group $\hat{\Gamma}$ of $\Gamma$ which are Fourier transforms of functions in $L^{1}(\Gamma)$ and $B(\hat{\Gamma})$ the algebra of functions on $\hat{\Gamma}$ which are Fourier-Stieltjes transforms of bounded regular Borel measures on $\Gamma$. A multiplier problem is to determine those complex-valued functions $\phi$ on $\hat{\Gamma}$ such that $\phi f \in B(\hat{\Gamma})$ whenever $f \in A(\hat{\Gamma})$. It is known that such functions are precisely the elements of $B(\hat{\Gamma})$ [10, Theorem 3.8.1].

To reach a more general situation, we reformulate the above problem in the following way. Let $M^{1}(\Gamma)$ be the Banach algebra of bounded regular Borel measures on $\Gamma$. Given a function $\phi$ as above define a map $m_{\phi}: L^{1}(\Gamma) \rightarrow M^{1}(\Gamma)$ by $m_{\phi} f=\mu$ where $\hat{\mu}(\gamma)=\phi(\gamma) \hat{f}(\gamma) ; \hat{f}$ is the Fourier transform of $f$, and $\hat{\mu}$ the Fourier-Stieltjes transform of $\mu$. It is easily seen that $m_{\phi}(g * f)=g * m_{\phi} f$ where $g \in L^{1}(\Gamma)$. Moreover using the closed graph theorem one can show that $m_{\phi}$ is continuous. Conversely if $m$ is a continuous linear map of $L^{1}(\Gamma)$ into $M^{1}(\Gamma)$ such that $m(g * f)=g * m f$ for $g$ in $L^{1}(\Gamma)$, then $g * m f=f * m g$ and it follows that there is a complex-valued function $\phi$ on $\hat{\Gamma}$ such that $m=m_{\phi}$. Now $L^{1}(\Gamma)$ is a Banach algebra and $M^{1}(\Gamma)$ is an $L^{1}(\Gamma)$-module and the multiplier problem is precisely the problem of determining the continuous module homomorphisms of $L^{1}(\Gamma)$ into $M^{1}(\Gamma)$.

Received by the editors February 2, 1971.

AMS 1970 subject classifications. Primary 43A15, 43A22, 46A05, 46H25; Secondary 22D99, 43A65.

Key words and phrases. Locally convex modules, tensor products, locally convex algebras, Banach modules, multipliers.

( ${ }^{1}$ This research was supported in part by the National Research Council of Canada.

Copyright (C) 1972, American Mathematical Society 
A more general multiplier problem may now be stated. Let $A$ be a Banach algebra, and $E$ and $F$ Banach $A$-modules. (A Banach $A$-module $E$ is a left $A$ module which is a Banach space and which satisfies $\|a x\|_{E} \leqq\|a\|_{A}\|x\|_{E}$.) The multiplier problem is that of determining the continuous module homomorphisms of $E$ into $F$. As a final step one can suppose only that $A$ is a topological algebra and $E$ and $F$ are locally convex $A$-modules in the sense of [9].

In recent years a large amount of work has been done on special cases of the above problem. (See, for example, [3], [4], [5], [6] and [8].) This indicates a need for general techniques which unify these results. The first steps in this direction have been made by M. A. Rieffel [7], [8] by the introduction of a suitable tensor product of Banach modules. Rieffel obtained the fundamental equation

$$
\operatorname{Hom}_{A}\left(F, E^{\prime}\right) \cong\left(E \otimes_{A} F\right)^{\prime}
$$

(see [7] for the definition of $E \otimes_{A} F$ ). By proving general representation theorems for $E \otimes_{A} F$ one can then obtain representation theorems for multipliers from a Banach module to the normed dual of another Banach module.

In this work we consider locally convex spaces which are modules over a topological algebra $A$ and we show that for a given polar topology $\Xi$ on $E^{\prime}$ there is a locally convex topology $\tau(\Im)$ on the tensor product $E \otimes_{A} F$ such that

$$
\operatorname{Hom}_{A}\left(F, E_{\Xi}^{\prime}\right)=\left(\left(E \otimes_{A} F\right)_{\tau}\right)^{\prime} .
$$

This gives a method for representing multipliers between locally convex $A$ modules. By obtaining representation theorems for $E \otimes_{A} F$ we are able to give rather easy proofs of a number of known results on multipliers as well as some new ones. However the main purpose of this paper is to develop a general framework for the study of multipliers, and the fact that we obtain some new results is some indication of the merit of this general approach.

The paper is organized as follows. $\$ 2$ contains the general results and definitions needed throughout the paper. Also in this section we give some representation theorems for $E \otimes_{A} A$ for the case in which $A$ has a unit or an approximate unit. In $\S 3$ we prove our main representation theorems. The final section is devoted to applications. In addition to some new results, we obtain easy proofs of results in [3], [4], [6] and [8].

2. General results. We begin by recalling some of the basic definitions and facts used in [9]. A topological algebra $A$ is a linear associative algebra over the complex field $C$ which is a topological vector space in which the maps $a \rightarrow a b$ and $a \rightarrow b a$ are continuous for each $b \in A$. A locally convex algebra is a topological algebra which is a locally convex (Hausdorff) space.

Definition. Let $A$ be a topological algebra. A locally convex left $A$-module $E$ is a locally convex space which is a left $A$-module such that the map $(a, x) \rightarrow a x$ of $A \times E$ into $E$ also satisfies 
1. for each $a \in A$, the map $x \rightarrow a x$ is a continuous map of $E$ into itself,

2. for each $x \in E$, the map $a \rightarrow a x$ is a continuous map of $A$ into $E$.

A locally convex right $A$-module is defined in the analogous fashion.

Let $A$ be a topological algebra, $E$ a locally convex right $A$-module, $F$ a locally convex left $A$-module, and $G$ a locally convex space. A bilinear map $f$ of $E \times F$ into $G$ is called $A$-balanced if $f(x a, y)=f(x, a y)$ for any $a \in A, x \in E, y \in F . B(E, F, G)$ is the set of all $A$-balanced bilinear maps. We shall write $B(E, F)$ in place of $B(E, F, C)$.

For each pair $(x, y) \in E \times F$, the map $f \rightarrow f(x, y)$ is a linear form on $B(E, F)$ and hence is an element $x \otimes y$ of the algebraic dual $B(E, F)^{*}$. The map

$$
\chi:(x, y) \rightarrow x \otimes y
$$

of $E \times F$ into $B(E, F)^{*}$ is bilinear and $A$-balanced. The linear span of $\chi(E \times F)$ in $B(E, F)^{*}$ is called the tensor product of $E$ and $F$ and is written $E \otimes_{A} F$.

Now let $\Im$ be a set of bounded subsets of $E$ such that $\cup \subseteq$ is total in $E$, and let $E_{\Im}^{\prime}$ be the dual of $E$ given the polar topology of sets in $\Im$. If for any $S$ in $\Im$ and any 0 -neighborhood $V$ in $E$ there is a 0 -neighborhood $W$ in $A$ such that $x \in S$ and $a \in W$ imply $x a \in V$, then we shall say that the action of $A$ on $E$ is $S$-hypocontinuous.

Proposition 1. Suppose that the action of $A$ on $E$ is $\widetilde{S}$-hypocontinuous. Then $E_{\Im}^{\prime}$ is a locally convex left A-module and there is a locally convex topology $\tau$ on $E \otimes_{A} F$ such that $\left(\left(E \otimes_{A} F\right)_{\tau}\right)^{\prime} \cong \operatorname{Hom}_{A}\left(F, E_{\Xi}^{\prime}\right)$ via an isomorphism $\psi$ such that $\langle\psi m(y), x\rangle$ $=\langle m, x \otimes y\rangle$ for $m \in\left(\left(E \otimes_{A} F\right)_{\tau}\right)^{\prime}$ and $x \in E, y \in F$.

Proof. The first assertion follows from Proposition 3 of [9], the remaining assertions are consequences of Theorem 2 of [9].

Whenever $\subseteq$ is the set of finite subsets of $E$ we write $E_{\sigma}^{\prime}$ in place of $E_{\Im}^{\prime}$ and the topology $\tau$ is called the inductive topology and is denoted by " $\iota$ ".

The problem now is to represent $\left(E \otimes_{A} F\right)_{\tau}$, and the remainder of this paper is devoted to doing this for special cases. We begin with a lemma which will be used several times in the sequel. We first recall from [9, Theorem 1] that if $\theta$ is an $A$ balanced $\subseteq$-hypocontinuous bilinear map $E \times F \rightarrow G$ [1; Chapitre III, $\$ 4$, No. 2], then there is a unique continuous linear map $\Theta:\left(E \otimes_{A} F\right)_{\tau} \rightarrow G$ such that $\Theta(x \otimes y)$ $=\theta(x, y)$.

LEMMA 1. Let $G$ be a locally convex space and $\theta$ an A-balanced 5 -hypocontinuous bilinear map $E \times F \rightarrow G$. Let $\Theta$ be the continuous linear map $\left(E \otimes_{A} F\right)_{\tau} \rightarrow G$ such that $\Theta(x \otimes y)=\theta(x, y)$. If for any finite subsets $x_{1}, x_{2}, \ldots, x_{n} \in E$ and $y_{1}$, $y_{2}, \ldots, y_{n} \in F$ such that $\sum_{i=1}^{n} \theta\left(x_{i}, y_{i}\right)=0$ we have $\sum_{i=1}^{n}\left\langle m y_{i}, x_{i}\right\rangle=0$ for all $m \in$ $\operatorname{Hom}_{A}\left(F, E_{\circlearrowleft}^{\prime}\right)$, then $\Theta$ is a continuous injection.

Proof. To show that $\Theta$ is one-one it is sufficient to show that for each continuous linear functional $u^{\prime}$ on $\left(E \otimes_{A} F\right)_{\tau}$ there is a linear functional $v^{\prime}$ on $\Theta\left(E \otimes_{A} F\right)$ such 
that $\left\langle u^{\prime}, u\right\rangle=\left\langle v^{\prime}, \Theta(u)\right\rangle$ for all $u \in E \otimes_{A} F$. Let $m \in \operatorname{Hom}_{A}\left(F, E_{\Im}^{\prime}\right)$ be such that $\langle m(y), x\rangle=\left\langle u^{\prime}, x \otimes y\right\rangle$ (Proposition 1), then define $v^{\prime}$ on $\Theta\left(E \otimes_{A} F\right.$ ) by

$$
\left\langle v^{\prime}, v\right\rangle=\sum_{i=1}^{n}\left\langle m\left(y_{i}\right), x_{i}\right\rangle
$$

where $v=\sum_{i=1}^{n} \theta\left(x_{i}, y_{i}\right)$. The hypotheses of the lemma imply that $v^{\prime}$ is well defined. Clearly, $v^{\prime}$ is a linear functional, moreover, $\left\langle u^{\prime}, u\right\rangle=\left\langle v^{\prime}, \Theta(u)\right\rangle$ for all $u \in E \otimes_{A} F$, and this completes the proof.

For the remainder of this section $A$ is a locally convex algebra. We shall also consider $A$ as a locally convex left $A$-module, and our concern now is to establish representation theorems for $E \otimes_{A} A$. The proof of the following proposition is immediate from the proof of Proposition 5 of [9]. The reader, however, can easily prove this for himself after reading the proof of Proposition 4 below.

Proposition 2. Suppose that $A$ has a unit and the action of $A$ on $E$ is $\subseteq$-hypocontinuous. Then $\left(E \otimes_{A} A\right)_{\tau} \cong E$.

When $A$ does not have a unit then it need not be true that $\left(E \otimes_{A} A\right)_{\tau} \cong E$. To proceed we use the following definition.

Definition. The essential part $E_{e}$ of a right $A$-module $E$ is the linear span of $E A$ in $E . E$ is called essential if $E_{e}$ is dense in $E$.

The next proposition gives a useful sufficient condition on $A$ for which the natural map $\left(E \otimes_{A} A\right)_{\tau}$ into $E_{e}$ is a continuous isomorphism.

Proposition 3. Suppose that the action of $A$ on $E$ is $\mathbb{S}$-hypocontinuous, and that for any subsets $a_{1}, a_{2}, \ldots, a_{n}$ of $A$ and $a_{1}^{\prime}, a_{2}^{\prime}, \ldots, a_{n}^{\prime}$ of $A^{\prime}$ such that $\sum_{i=1}^{n} a_{i}^{\prime} a_{i}=0$ we have $\sum\left\langle a_{i}, a_{i}^{\prime}\right\rangle=0$. Then there is a continuous isomorphism of $\left(E \otimes_{A} A\right)_{\tau}$ onto $E_{e}$.

Proof. Let $\Theta:\left(E \otimes_{A} A\right)_{\tau} \rightarrow E_{e}$ be the continuous linear map satisfying $\Theta(x \otimes a)$ $=x a$. We shall use Lemma 1 to prove the result. Accordingly let $m \in \operatorname{Hom}_{A}\left(A, E_{\Xi}^{\prime}\right)$ and let $a_{1}, a_{2}, \ldots, a_{n}$ and $x_{1}, x_{2}, \ldots, x_{n}$ be finite subsets of $A$ and $E$ such that $\sum x_{i} a_{i}=0$. Then for any $a \in A$,

$$
\begin{aligned}
0 & =\sum\left\langle x_{i} a_{i}, m a\right\rangle=\sum\left\langle x_{i}, a_{i} m a\right\rangle \\
& =\sum\left\langle x_{i}, m a_{i} a\right\rangle=\sum\left\langle m^{\prime} x_{i}, a_{i} a\right\rangle=\sum\left\langle\left(m^{\prime} x_{i}\right) a_{i}, a\right\rangle .
\end{aligned}
$$

Thus $\sum\left(m^{\prime} x_{i}\right) a_{i}=0$, so by hypothesis $\sum\left\langle x_{i}, m a_{i}\right\rangle=\sum\left\langle m^{\prime} x_{i}, a_{i}\right\rangle=0$. This completes the proof.

Definition. A net $\left(u_{j}: j \in J\right)$ in a locally convex algebra $A$ is called a weak right approximate unit if for each $a \in A, a^{\prime} \in A^{\prime}$ we have

$$
\lim _{j}\left\langle a u_{j}, a^{\prime}\right\rangle=\left\langle a, a^{\prime}\right\rangle .
$$

A weak right approximate unit is called bounded if it is a bounded subset of $A$.

Note that if $A$ has a weak right approximate unit then $A$ satisfies the hypotheses of Proposition 3. If $A$ has a bounded weak right approximate unit then more is true. 
Proposition 4. Suppose that $E$ is barreled and that $A$ has a bounded weak right approximate unit. If the action of $A$ on $E$ is $\Xi$-hypocontinuous, then $\left(E \otimes_{A} A\right)_{\tau} \cong E_{e}$.

Proof. Let $\Theta$ be as in the proof of Proposition 3. By that proposition it is sufficient now to show that $\Theta(H)$ is an equicontinuous subset of $\left(\left(E \otimes_{A} A\right)_{\tau}\right)^{\prime}$ only if $H$ is an equicontinuous subset of $\left(E_{e}\right)^{\prime}$. By Theorem 1 of [9],

$$
\Theta^{\prime}(H) \circ \chi=\left\{\Theta^{\prime}(h) \circ \chi: h \in H\right\}
$$

is an S-equihypocontinuous subset of $B(E, A)(\chi$ is the canonical map $E \times A \rightarrow$ $\left.E \otimes_{A} A\right)$. Since $E$ is barreled, $\Theta^{\prime}(H) \circ \chi$ is a $\beta$-equihypocontinuous subset of $B(E, A)$ where $\beta$ is the set of bounded subsets of $A$ [1, Chapitre 3, $\$ 4$, Proposition 6]. Let $\left(u_{j}: j \in J\right)$ be a bounded weak approximate unit in $A$, then given $\varepsilon>0$ there is a 0 -neighborhood $V$ in $E$ such that $x \in V$ implies

$$
\left|\Theta^{\prime}(h) \circ \chi\left(x, u_{j}\right)\right| \leqq \varepsilon / 2 \text { for all } j \in J .
$$

Let $W=V \cap E_{e}$, then for $x \in W$ and $h \in W$ there is a $j_{0}$ such that $j \geqq j_{0}$ implies $\left|\left\langle x-x u_{j}, h\right\rangle\right| \leqq \varepsilon / 2$ since the map $a \rightarrow x a$ is continuous with respect to the $\sigma\left(A, A^{\prime}\right)$ and $\sigma\left(E, E^{\prime}\right)$-topologies. Thus if $x \in W$ and $h \in H$ we have

$$
|\langle x, h\rangle| \leqq\left|\left\langle x-x u_{j}, h\right\rangle\right|+\left|\left\langle x u_{j}, h\right\rangle\right| \leqq \varepsilon / 2+\left|\Theta^{\prime}(h) \circ \chi\left(x, u_{j}\right)\right| \leqq \varepsilon .
$$

Thus $H$ is equicontinuous and this completes the proof.

Corollary. Let $E$ and $A$ be as in the theorem, and let $E \tilde{\otimes}_{A} A$ be the completion of $\left(E \otimes_{A} A\right)_{\tau}$. If $E$ is complete and essential, then $E \tilde{\otimes}_{A} A \cong E$.

3. The main theorems. In this section we establish our main results. Much of the remainder of the paper is devoted to consequences of these results. The setting is the following: $A$ is a topological algebra $E=$ ind $\lim E_{\alpha}$ and $F=\operatorname{ind} \lim F_{\alpha}$ are locally convex right and left $A$-modules respectively which as locally convex spaces are inductive limits of metrizable spaces, we suppose also that $E_{\alpha}$ (or the $F_{\alpha}$ ) are barreled. $G$ is a complete locally convex space and $\theta$ is a separately continuous $A$ balanced bilinear map of $E \times F$ into $G$. For each $\alpha$ let $\left(p_{n}^{\alpha}\right)$ and $\left(q_{n}^{\alpha}\right)$ be directed sets of seminorms generating the topology of $E_{\alpha}$ and $F_{\alpha}$. For each $\alpha$ let

$$
\begin{aligned}
H_{\alpha}=\left\{u \in G: u=\sum_{i=1}^{\infty} \theta\left(x_{i}, y_{i}\right), \sum_{i} p_{n}^{\beta}\left(x_{i}\right) q_{n}^{\beta}\left(y_{i}\right)\right. & <\infty, \text { all } n, \\
\beta & \left.\geqq \alpha \text { and } x_{i} \in E_{\alpha}, y_{i} \in F_{\alpha}\right\} .
\end{aligned}
$$

For each $n$ and $\alpha$ we define a seminorm $r_{n}^{\alpha}$ on $H_{\alpha}$ by

$$
r_{n}^{\alpha}(u)=\inf \left\{\sum_{i=1}^{\infty} p_{n}^{\alpha}\left(x_{i}\right) q_{n}^{\alpha}\left(y_{i}\right): u=\sum \theta\left(x_{i}, y_{i}\right)\right\} .
$$

We give each $H_{\alpha}$ the topology generated by $\left(r_{n}^{\alpha}\right)$. 
Proposition 5. Let $V_{n}^{\alpha}=\left\{x \in E_{\alpha}: p_{n}^{\alpha}(x) \leqq 1\right\}$ and $W_{n}^{\alpha}=\left\{y \in F_{\alpha}: q_{n}^{\alpha}(y) \leqq 1\right\}$ then $r_{n}^{\alpha}$ is the gauge of $\mathrm{cl} \operatorname{co} \theta\left(V_{n}^{\alpha} \times W_{n}^{\alpha}\right)$, the closure in $H_{\alpha}$ of the circled convex hull of $\theta\left(V_{n}^{\alpha} \times W_{n}^{\alpha}\right)$.

Proof. We argue as in [11, Chapter III, §6.3]. Let $N=\left\{u: r_{n}^{\alpha}(u)<1\right\}$ and $M=\left\{u: r_{n}^{\alpha}(u) \leqq 1\right\}$, it is sufficient to show

$$
N \subset \operatorname{cl} \operatorname{co} \theta\left(V_{n}^{\alpha} \times W_{n}^{\alpha}\right) \subset M .
$$

Clearly $\theta\left(V_{n}^{\alpha} \times W_{n}^{\alpha}\right) \subset M$ and since $M$ is closed and convex we have

$$
\mathrm{cl} \operatorname{co} \theta\left(V_{n}^{\alpha} \times W_{n}^{a}\right) \subset M \text {. }
$$

Now suppose $u \in N$, then $u=\sum \theta\left(x_{i}, y_{i}\right)$ with $\sum p_{n}^{\alpha}\left(x_{i}\right) q_{n}^{\alpha}\left(y_{i}\right)<1$. There are real numbers $\varepsilon_{i}>0$ such that if $\beta_{i}=\left(p_{n}^{\alpha}\left(x_{i}\right)+\varepsilon_{i}\right)\left(q_{n}^{\alpha}\left(y_{i}\right)+\varepsilon_{i}\right)$, then $\sum \beta_{i}<1$. Put $v_{i}=x_{i} / p_{n}^{\alpha}\left(x_{i}\right)$ $+\varepsilon_{i}$ and $w_{i}=y_{i} / q_{n}^{\alpha}\left(y_{i}\right)+\varepsilon_{i}$, then $v_{i} \in W_{n}^{\alpha}$ and $u=\sum \beta_{i} \theta\left(v_{i}, w_{i}\right) \in \operatorname{cl} \operatorname{co} \theta\left(V_{n}^{\alpha} \times W_{n}^{\alpha}\right)$. This completes the proof.

From Proposition 5 and the continuity of $\theta \mid E_{\alpha} \times F_{\alpha} \rightarrow G$ [1, Chapitre III, $\S 4$, Proposition 2] it follows that the injection $H_{\alpha} \rightarrow G$ is continuous. This coupled with the completeness of $G$ yields

Proposition 6. $H_{\alpha}$ is a Fréchet space.

Proof. We will only be concerned with showing completeness. Let $\left(u_{n}\right)$ be a Cauchy sequence in $H_{\alpha}$. Choose a subsequence $\left(v_{n}\right) \subset\left(u_{n}\right)$ such that $r_{n}^{\alpha}\left(v_{n+1}-v_{n}\right)$ $\leqq 1 / 2^{n}$. Then $\left(v_{n}\right)$ is Cauchy in the original topology of $G$ so there is a $v$ such that $v=\lim v_{n}$ in $G$. It remains to show that $v=\lim v_{n}$ in $H_{\alpha}$. For $m \geqq n$ we have

$$
r_{n}^{\alpha}\left(v-v_{m}\right)=r_{n}^{\alpha}\left(\sum_{j \geqq m} v_{j+1}-v_{j}\right) \leqq \sum_{j} r_{n}^{\alpha}\left(v_{j+1}-v_{j}\right) \leqq \sum_{j} r_{j}^{\alpha}\left(v_{j+1}-v_{j}\right) \leqq \sum_{j} 1 / 2^{j} .
$$

And this completes the proof.

For $\alpha \leqq \beta$ the injection $H_{\alpha} \rightarrow H_{\beta}$ is continuous, thus the locally convex inductive limit $H=$ ind $\lim H_{\alpha}$ exists. While it is true that $\theta \mid E_{\alpha} \times F_{\alpha}$ is a continuous map into $H$ one cannot conclude that $\theta$ is continuous from $E \times F$ into $H$ since one does not in general have $E \times F=$ ind $\lim E_{\alpha} \times F_{\alpha}$ as topological spaces. However all we need is the separate continuity of $\theta$ and this we can demonstrate.

Propositron 7. $\theta: E \times F \rightarrow H=$ ind $\lim H_{\alpha}$ is separately continuous.

Proof. Given $y$ in $F$ let $\theta_{y}$ be the map $x \rightarrow \theta(x, y)$. By the definition of the inductive topology it is sufficient to show that there is an $\alpha_{0}$ such that $\alpha \geqq \alpha_{0}$ implies $\theta_{y} \mid E_{\alpha}$ is continuous from $E_{\alpha}$ into $H$. Choose $\alpha_{0}$ such that $y \in F_{\alpha_{0}}$, then $y \in F_{\alpha}$ for every $\alpha \geqq \alpha_{0}$, and it follows from Proposition 5 that $\theta_{y} \mid E_{\alpha}$ is continuous into $H_{\alpha}$, and hence into $H$. An analogous argument works for the continuity of $y \rightarrow \theta(x, y)$ for each $x$ in $E$.

From Proposition 7 above and Theorem 1 of [9] we have the existence of a continuous linear map $\Theta:\left(E \otimes_{A} F\right)_{\iota} \rightarrow H$ such that $\Theta(x \otimes y)=\theta(x, y)$. 
TheOREM 1. Let E, F, H, $\theta$ and $\Theta$ be as above. The following conditions are equivalent:

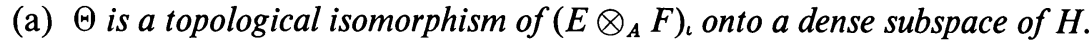

(b) For sequences $\left(x_{n}\right) \subset E_{\alpha}$ and $\left(y_{n}\right) \subset F_{\alpha}$ such that $\sum \theta\left(x_{i}, y_{i}\right)=0$ and $\sum p_{n}^{\beta}\left(x_{i}\right) q_{n}^{\beta}\left(y_{i}\right)$

$<\infty$ for every $n$ and $\beta \geqq \alpha$ we have $\sum\left\langle x_{i}, m y_{i}\right\rangle=0$ for every $m \in \operatorname{Hom}_{A}\left(F, E_{\sigma}^{\prime}\right)$.

(c) For any finite subsets $x_{1}, x_{2}, \ldots, x_{n}$ of $E$ and $y_{1}, y_{2}, \ldots, y_{n}$ of $F$ such that $\sum \theta\left(x_{i}, y_{i}\right)=0$ we have $\sum\left\langle x_{i}, m y_{i}\right\rangle=0$ for every $m \in \operatorname{Hom}_{A}\left(F, E_{\sigma}^{\prime}\right)$.

Proof. We begin by showing (a) implies (b). If (a) is true, then the map $\Theta^{\prime}$ is an isomorphism of $H^{\prime}$ onto $\left(E \otimes_{A} F\right)_{\text {c }}^{\prime}$ with the property that for any $m \in \operatorname{Hom}_{A}\left(F, E_{\sigma}^{\prime}\right)$ there is a $u^{\prime} \in H^{\prime}$ such that the equation $\left\langle u^{\prime}, \Theta(x \otimes y)\right\rangle=\langle x, m y\rangle$ holds for $x \in E$ and $y \in F$ (Proposition 1). Now letting $u_{n}=\sum_{m=1}^{n} \theta\left(x_{i}, y_{i}\right)$ where $\left(x_{i}\right) \subset E_{\alpha}$ and $\left(y_{i}\right) \subset F_{\alpha}$ satisfy the condition of (b) we have $u_{n} \rightarrow 0$ in $H$. Consequently $\left\langle u^{\prime}, u_{n}\right\rangle \rightarrow 0$ and since $\left\langle u^{\prime}, u_{n}\right\rangle=\sum_{i=1}^{n}\left\langle x_{i}, m y_{i}\right\rangle$ we have that $\sum_{i=1}^{\infty}\left\langle x_{i}, m y_{i}\right\rangle=0$.

Clearly (b) implies (c) so we suppose now that (c) holds. By Lemma 1 we have that $\Theta$ is one-one. From Proposition 5 it is clear that $\theta\left(E_{\alpha} \times F_{\alpha}\right)$ is total in $H_{\alpha}$ and from this we see that $\Theta\left(E \otimes_{A} F\right)$ is dense in $H$. Let $M$ be a subset of $H^{\prime}$, to show that $\Theta$ is a topological isomorphism it is sufficient to show that the equicontinuity of $\Theta^{\prime}(M)$ implies that $M$ is equicontinuous. If $\Theta^{\prime}(M)$ is equicontinuous in $\left(E \otimes_{A} F\right)^{c}$ then by Theorem 1 of [9], $\Theta^{\prime}(M) \circ \chi=\left\{\Theta^{\prime} u^{\prime} \circ \chi: u^{\prime} \in M\right\}$ ( $\chi$ is the canonical map of $E \times F$ into $\left.\left(E \otimes_{A} F\right)_{\iota}\right)$ is a separately equicontinuous subset of bilinear maps. Thus since either $E_{\alpha}$ or $F_{\alpha}$ is barreled we have by [1, Chapitre III, $\S 4$, Proposition 10] that $\Theta^{\prime}(M) \circ \chi \mid E_{\alpha} \times F_{\alpha}$ is an equicontinuous set of bilinear forms, thus there are 0-neighborhoods $V_{\alpha}, W_{\alpha}$ in $E_{\alpha}$ and $F_{\alpha}$ such that $x \in V_{\alpha}, y \in W_{\alpha}$ and $u^{\prime} \in M$ imply

$$
\left|\left\langle u^{\prime}, \theta(x, y)\right\rangle\right|=\left|\Theta^{\prime} u^{\prime} \circ \chi(x, y)\right| \leqq 1 .
$$

If $u \in \operatorname{cl} \operatorname{co} \theta\left(V_{\alpha} \times W_{\alpha}\right)$ then $\left|\left\langle u^{\prime}, u\right\rangle\right| \leqq 1$. By Proposition 5, cl co $\theta\left(V_{\alpha} \times W_{\alpha}\right)$ is a neighborhood for each $\alpha$ and this implies that $M$ is equicontinuous.

If we apply the above theorem to the situation in which $F=A, G=E$ and $\theta(x, a)$ $=x a$, we can argue as in the proof of Proposition 3 and obtain the following:

THEOREM 2. Suppose that $A$ is a locally convex algebra and that for any subsets $a_{1}, a_{2}, \ldots, a_{n}$ of $A$ and $a_{1}^{\prime}, a_{2}^{\prime}, \ldots, a_{n}^{\prime}$ of $A^{\prime}$ such that $\sum a_{i} a_{i}^{\prime}=0$ we have $\sum\left\langle a_{i}, a_{i}^{\prime}\right\rangle=0$. Then $\left(E \otimes_{A} A\right)_{\text {، }}$ is topologically isomorphic to a dense subspace of $H$.

The above two theorems have some interesting consequences. We will in the next section derive a number of known and new results from them. We do not think this exhausts all possibilities.

4. Applications. We begin this section by using Theorem 2 to generalize a result of Gaudry [4]. Let $\Gamma$ be a locally compact group, $\mathscr{K}(\Gamma)$ the space of continuous complex-valued functions on $\Gamma$ having compact support, and for each compact subset $K$ of $\Gamma$ let $\mathscr{K}(\Gamma, K)$ be the subspace of $\mathscr{K}(\Gamma)$ of functions whose support is in 
$K$. Each $\mathscr{K}(\Gamma, K)$ is a Banach space given the uniform norm, and we give $\mathscr{K}(\Gamma)$ the finest locally convex topology such that for each $K$ the injection $\mathscr{K}(\Gamma, K) \rightarrow \mathscr{K}(\Gamma)$ is continuous; i.e. $\mathscr{K}(\Gamma)=$ ind $\lim \mathscr{K}(\Gamma, K) . \mathscr{K}(\Gamma)$ is a topological algebra with the convolution product

$$
f * g\left(\gamma_{0}\right)=\int f\left(\gamma_{0} \gamma\right) g\left(\gamma^{-1}\right) d \gamma .
$$

For $f \in \mathscr{K}(\Gamma)$, let $f^{\sim}(\gamma)=f\left(\gamma^{-1}\right) \Delta\left(\gamma^{-1}\right)$. By $\mathscr{K}(\Gamma)^{r}$ we mean $\mathscr{K}(\Gamma)$ considered as a locally convex right $\mathscr{K}(\Gamma)$-module with the action of $\mathscr{K}(\Gamma)$ on $\mathscr{K}(\Gamma)^{r}$ given by $g \rightarrow f^{\sim} * g$. Our purpose now is to represent $\mathscr{K}(\Gamma)^{r} \otimes_{\mathscr{K}(\Gamma)} \mathscr{K}(\Gamma)$. We shall need the following lemma, which is probably not at all new.

LeMmA 2. Let $f \in \mathscr{K}(\Gamma)$ and $\mu \in M(\Gamma)=\mathscr{K}(\Gamma)^{\prime}$, then given any $\varepsilon>0$ there is an $h \in \mathscr{K}(\Gamma)$ such that $|\langle f, \mu\rangle-\langle f * h, \mu\rangle| \leqq \varepsilon$.

Proof. First suppose $|\mu|(\operatorname{Supp} f)=0$. Then we can find a relatively compact neighborhood $W$ of the identity in $\Gamma$ such that $|\mu|((\operatorname{Supp} f) W)<\varepsilon /\|f\|$. Then choosing $h \in \mathscr{K}(\Gamma)$ such that $\operatorname{Supp} h \subset W, h \geqq 0$ and $\int h\left(\gamma^{-1}\right) d \gamma=1$, we have $|\langle f * h, \mu\rangle|<\varepsilon$. Thus we may suppose $|\mu|(\operatorname{Supp} f)=\frac{1}{2}$. Let $U$ be an open relatively compact subset containing $\operatorname{Supp} f$, such that $|\mu|(U) \leqq \frac{3}{4}$ and let $V$ be an open set containing $U$ with $|\mu|(V) \leqq 1$. Choose $g \in \mathscr{K}(\Gamma)$ such that $g \mid U=1 ; 0 \leqq g \leqq 1$ and Supp $g \subset V$. Since $f$ is uniformly continuous there is a compact neighborhood $W$ of the identity of $\Gamma$ such that $\left|f(\gamma)-f\left(\gamma \gamma_{0}\right)\right| \leqq \varepsilon$ for all $\gamma \in \Gamma$ and $\gamma_{0} \in W$; $(\operatorname{Supp} f) W$ $\subset U$ and $W=W^{-1}$. It is simple to conclude that for $\gamma_{0} \in W$ we have $\left|f(\gamma)-f\left(\gamma \gamma_{0}\right)\right|$ $\leqq \varepsilon g(\gamma)$ for all $\gamma \in \Gamma$. Now choose $h \in \mathscr{K}(\Gamma)$ such that Supp $h \subset W, h \geqq 0$ and $\int h\left(\gamma^{-1}\right) d \gamma=1$. Then

$$
\begin{aligned}
|\langle f, \mu\rangle-\langle f * h, \mu\rangle| & =\left|\int f d \mu \int h\left(\gamma_{0}^{-1}\right) d \gamma_{0}-\iint f\left(\gamma \gamma_{0}\right) h\left(\gamma_{0}^{-1}\right) d \gamma_{0} d \mu(\gamma)\right| \\
& \leqq \iint\left|f(\gamma)-f\left(\gamma \gamma_{0}\right)\right| h\left(\gamma_{0}^{-1}\right) d|\mu|(\gamma) d \gamma_{0} \\
& \leqq \iint \varepsilon g(\gamma) h\left(\gamma_{0}^{-1}\right) d|\mu|(\gamma) d \gamma_{0} \leqq \varepsilon .
\end{aligned}
$$

To obtain a representation of $\mathscr{K}(\Gamma)^{r} \otimes_{\mathscr{K}(\Gamma)} \mathscr{K}(\Gamma)$, we apply Theorem 2 with the following changes in notation: $E=\mathscr{K}(\Gamma)^{r} ; E_{\alpha}=\mathscr{K}(\Gamma, K), A=\mathscr{K}(\Gamma) ; A_{\alpha}=$ $\mathscr{K}\left(\Gamma, K^{-1}\right)$ and $\theta(g, f)=f^{\sim} * g$. We put $D_{K}(\Gamma)=H_{\alpha}, D(\Gamma)=H$ and our notation conforms precisely to that of Gaudry [4].

THEOREM 3. $\mathscr{K}(\Gamma)^{r} \otimes_{\mathscr{K}_{(\Gamma)}} \mathscr{K}(\Gamma)$ is topologically isomorphic to a dense vector subspace of $D(\Gamma)$.

Proof. We show that the hypothesis of Theorem 2 is satisfied. Let $f_{1}, \ldots, f_{n}$ $\in \mathscr{K}(\Gamma)$ and $\mu_{1}, \ldots, \mu_{n} \in M(\Gamma)$ and suppose $\sum f_{i} \circ \mu_{i}=0$. Given any $\varepsilon>0$ there is, by Lemma $2, h_{i} \in \mathscr{K}(\Gamma)$ such that

$$
\left|\sum\left\langle f_{i}, \mu_{i}\right\rangle-\sum\left\langle f_{i} * h_{i}, \mu_{i}\right\rangle\right| \leqq \varepsilon .
$$


Since $\left\langle f_{i} * h_{i}, \mu_{i}\right\rangle=\left\langle h_{i}, f_{i} \circ \mu_{i}\right\rangle$ we have $\sum\left\langle f_{i}, \mu_{i}\right\rangle=0$, and this completes the proof.

In [4] Gaudry calls the elements of $D(\Gamma)^{\prime}$ quasimeasures and he defines a convolution product of $f \in \mathscr{K}(\Gamma)$ and $\sigma \in D(\Gamma)^{\prime}$ to be the element of $D(\Gamma)^{\prime}$ defined by $\langle g, f * \sigma\rangle=\langle f * g, \sigma\rangle$ for any $g \in D(\Gamma), f \in \mathscr{K}(\Gamma)$ [4, Lemma 2.9].

COROLlaRY. Let $m$ be a continuous linear map $\mathscr{K}(\Gamma) \rightarrow M(\Gamma)_{\sigma}$ satisfying

$$
m(g * f)=g * m f \text {. }
$$

Then there is a quasimeasure $\sigma \in D(\Gamma)^{\prime}$ such that

$$
m f=f * \sigma .
$$

Conversely given any $\sigma \in D(\Gamma)^{\prime}$, the equation $\left(\begin{array}{l}* \\ *\end{array}\right)$ defines a continuous linear map $\mathscr{K}(\Gamma) \rightarrow M(\Gamma)_{\sigma}$ satisfying $\left(^{*}\right)$.

Proof. By Theorem 3 and Proposition 1, we have $\operatorname{Hom}_{\mathscr{K}(\Gamma)}\left(\mathscr{K}(\Gamma), M(\Gamma)_{\sigma}\right)$ $=D(\Gamma)^{\prime}$.

REMARK. For locally compact abelian groups $G$, the above corollary is Gaudry's Theorem 3.2 of [4].

The representation of tensor products of other modules arising in harmonic analysis is often considerably easier than Theorem 3 above. The next theorem gives some of these. For a locally compact group $\Gamma$ let $C(\Gamma)$ be the space of continuous complex-valued functions given the compact-open topology. $C_{0}(\Gamma)$ is the subspace of $C(\Gamma)$ of functions which "vanish at infinity" taken with the usual norm topology. $M^{1}(\Gamma)=C_{0}(\Gamma)^{\prime}$ is the subspace of $M(\Gamma)$ of bounded measures and $M_{c}(\Gamma)=C(\Gamma)^{\prime}$ is the subspace $M(\Gamma)$ of measures having compact support. We give $M_{c}(\Gamma)$, the $\sigma\left(M_{c}(\Gamma), C(\Gamma)\right)$-topology. $\mathscr{K}(\Gamma)$ and $C(\Gamma)$ are locally convex right $M_{c}(\Gamma)$-modules and $C_{0}(\Gamma)$ is a locally convex right $M^{1}(\Gamma)$-module with action defined by $f \rightarrow f^{\sim} * \mu$ where in each case

$$
f * \mu\left(\gamma_{0}\right)=\int f\left(\gamma_{0} \gamma^{-1}\right) \Delta\left(\gamma^{-1}\right) d \mu(\gamma) .
$$

THEOREM 4. Let $\Gamma$ be a locally compact group. Then

(a) $\left(\mathscr{K}(\Gamma) \otimes_{M_{c}(\Gamma)} M_{c}(\Gamma)\right)_{\imath} \cong \mathscr{K}(\Gamma)$,

(b) $\left(C(\Gamma) \otimes_{M_{c}(\Gamma)} M_{c}(\Gamma)\right)_{\iota} \cong C(\Gamma)$,

(c) $\left(C_{0}(\Gamma) \otimes_{M^{1}(\Gamma)} M^{1}(\Gamma)\right)_{\imath} \cong C_{0}(\Gamma)$.

Proof. These are all immediate consequences of Proposition 2 of $\$ 2$.

If $m$ is a mapping of some subspace of $C(\Gamma)$ into $C(\Gamma)$, then we say that $m$ commutes with translations if $m(\gamma f)=\gamma m f$ for all $\gamma \in \Gamma$, where $\gamma f$ is defined by $\gamma f\left(\gamma_{0}\right)=f\left(\gamma^{-1} \gamma_{0}\right)$

COROllary (BRAINERD AND EdWARDS [3]). Let $\Gamma$ be a locally compact group. Then

(a) If $m: \mathscr{K}(\Gamma) \rightarrow C(\Gamma)$ is linear, continuous and commutes with translations, then there is a $\mu \in M(\Gamma)$ such that $m f=f * \mu$. 
(b) If $m: C(\Gamma) \rightarrow C(\Gamma)$ is linear, continuous and commutes with translations, then there is $a \mu \in M_{c}(\Gamma)$ such that $m f=f * \mu$.

(c) If $m: C_{0}(\Gamma) \rightarrow C_{0}(\Gamma)$ is linear, continuous and commutes with translations, then there is a $\mu \in M^{1}(\Gamma)$ such that $m f=f * \mu$.

Proof. $m$ satisfies (a) if and only if its adjoint $m^{\prime}$ is in $\operatorname{Hom}_{M_{c}(\Gamma)}\left(M_{c}(\Gamma), M(\Gamma)_{\sigma}\right)$. Similarly for (b) and (c).

Definition. Let $A$ be a Banach algebra. A Banach left $A$-module $E$ is a left $A$-module such that for $a \in A$ and $x \in E,\|a x\| \leqq\|a\|\|x\|$.

We now consider the $L^{p}(\Gamma)$ spaces where $\Gamma$ is a locally compact group. For $1 \leqq p \leqq \infty$, these are Banach left $L^{1}(\Gamma)$-modules with the action of $L^{1}(\Gamma)$ on $L^{p}(\Gamma)$ given by $g \rightarrow f * g$. For $1<p \leqq \infty, L^{p}(\Gamma)$ is the dual module of $L^{q}(\Gamma), 1 \leqq q<\infty$, $1 / p+1 / q=1$ and as such is a Banach right $L^{1}(\Gamma)$-module. Let $L_{c}^{1}(\Gamma)$ be the subset of $L^{1}(\Gamma)$ consisting of elements having compact support and let $L_{c, K}^{1}(\Gamma)(K \subset \mathrm{I}, K$ compact) be the subspace of $L_{c}^{1}(\Gamma)$ of elements whose support is in $K$. We give $L_{c}^{1}(\Gamma)$ the finest locally convex topology such that the maps $L_{c, K}^{1}(\Gamma) \rightarrow L_{c}^{1}(\Gamma)$ are continuous: $L_{c}^{1}(\Gamma)$ with this topology is a locally convex algebra.

THEOREM 5. Let $\beta$ be the set of bounded subsets of $L^{p}(\Gamma), 1<p<\infty$, and let $\bar{L}^{p}(\Gamma) \tilde{\otimes}_{L_{c}^{1}(\Gamma)} L_{c}^{1}(\Gamma)$ be the completion of $\left(\bar{L}^{p}(\Gamma) \otimes_{L_{c}^{1}(\Gamma)} L_{c}^{1}(\Gamma)\right)_{\tau(\beta)}$. Then

$$
\bar{L}^{p}(\Gamma) \tilde{\otimes}_{L_{c}^{1}(\Gamma)} L_{c}^{1}(\Gamma) \cong L^{p}(\Gamma) .
$$

Proof. This follows from Proposition 4. We leave the details to the reader.

Corollary (Brainerd AND Edwards [3]). Suppose $1<p<\infty$ and that $m: L_{c}^{1}(\Gamma)$ $\rightarrow L^{p}(\Gamma)$ is linear, continuous and commutes with translations. Then there is an $f \in L^{p}(\Gamma)$ such that $m g=g * f$.

Now let $A$ be a Banach algebra, $E$ a Banach right $A$-module and $F$ a Banach left $A$-module. In this case every separately continuous bilinear map of $E \times F$ into a Banach space $G$ is continuous and hence all the topologies $\tau$ on $E \otimes_{A} F$ coincide with the finest locally convex topology $\pi$ on $E \otimes_{A} F$ such that the canonical map $\chi: E \times F \rightarrow E \otimes_{A} F$ is continuous. This topology is normable, and a suitable norm on $E \otimes_{A} F$ is given by

$$
\|u\|=\inf \left\{\sum_{i=1}^{n}\left\|x_{i}\right\|\left\|y_{i}\right\|: u=\sum x_{i} \otimes y_{i}\right\} .
$$

Proposition 8. The completion $E \tilde{\otimes}_{A} F$ of $\left(E \otimes_{A} F\right)_{\pi}$ is a Banach space having the following property: for each A-balanced bounded bilinear map $\theta$ of $E \times F$ into a Banach space $G$, there is a bounded linear map $\Theta: E \tilde{\otimes}_{A} F \rightarrow G$ such that $\Theta(x \otimes y)$ $=\theta(x, y)$ and $\|\Theta\|=\|\theta\|$.

Proof. From Theorem 1 of [9], and the well-known property of the completion of a normed space we obtain the existence of $\Theta$. It remains now to show $\|\Theta\|=\|\theta\|$. Clearly we have $\|\theta\| \leqq\|\Theta\|$. Let $u \in E \tilde{\otimes}_{A} F$ and suppose $u=\sum x_{i} \otimes y_{i}$, then $\|\Theta(u)\| \leqq\|\theta\| \sum\left\|x_{i}\right\|\left\|y_{i}\right\|$ and hence $\|\Theta\|=\|\theta\|$. 
Remark. The above proposition and Theorem 2.5 of [7] show that $E \tilde{\otimes}_{A} F$ is isometric and isomorphic to the tensor product used by Rieffel in [7] and [8].

The representation theorems of $\S 3$ becorne for Banach modules the following

TheOREM 6. Let $\theta$ be an A-balanced bilinear map of $E \times F$ into a Banach space $G$. Let

$$
H=\left\{u \in G: u=\sum_{1}^{\infty} \theta\left(x_{i}, y_{i}\right), \sum\left\|x_{i}\right\|\left\|y_{i}\right\|<\infty\right\} .
$$

Then with respect to the norm

$$
u \rightarrow\|u\|=\inf \left\{\sum_{1}^{\infty}\left\|x_{i}\right\|\left\|y_{i}\right\|: u=\sum_{1}^{\infty} \theta\left(x_{i}, y_{i}\right)\right\}
$$

$H$ is a Banach space and the following conditions are equivalent:

(a) $\Theta: E \tilde{\otimes}_{A} F \rightarrow H$ is a norm isomorphism.

(b) For sequences $\left(x_{n}\right) \subset E$ and $\left(y_{n}\right) \subset F$ such that $\sum \theta\left(x_{n}, y_{n}\right)=0$ and $\sum\left\|x_{n}\right\|\left\|y_{n}\right\|$ $<\infty$ we have $\sum\left\langle x_{n}, m y_{n}\right\rangle=0$ for every $m \in \operatorname{Hom}_{A}\left(F, E_{\beta}^{\prime}\right)$.

(c) For any finite subsets $x_{1}, x_{2}, \ldots, x_{n}$ of $E$ and $y_{1}, y_{2}, \ldots, y_{n}$ of $F$ such that $\sum \theta\left(x_{i}, y_{i}\right)=0$ we have $\sum\left\langle x_{i}, m y_{i}\right\rangle=0$ for every $m \in \operatorname{Hom}_{A}\left(F, E_{\beta}^{\prime}\right)$.

Proof. This follows from Theorem 1 and the well-known properties of the completion of a normed space.

For $F=A, G=E$ and $\theta(x, a)=x a$ we obtain as in $\S 3$ :

THEOREM 7. If for subsets $a_{1}, a_{2}, \ldots, a_{n}$ of $A$ and $a_{1}^{\prime}, a_{2}^{\prime}, \ldots, a_{n}^{\prime}$ of $A^{\prime}$ such that $\sum a_{i} a_{i}^{\prime}=0$ we have $\sum\left\langle a_{i}, a_{i}^{\prime}\right\rangle=0$, then $E \tilde{\otimes}_{A} A$ is norm isomorphic to $H$.

Now let $E=A$ so that $H$ becomes the space which Máté denotes by $Y^{\prime}$ in [6], and $A^{\prime}$ the dual of $A$ with the norm topology.

Corollary (MÁtÉ). Let $A$ be a Banach algebra satisfying the condition of Theorem 7, then $\operatorname{Hom}_{A}(A, A)$ is norm isomorphic to a subspace of $H^{\prime}$.

Proof. Observe that

$$
\operatorname{Hom}_{A}(A, A) \subset \operatorname{Hom}_{A}\left(A, A^{\prime \prime}\right)=\left(A^{\prime} \otimes_{A} A\right)^{\prime}=Y^{\prime} .
$$

Theorem 6 is closely related to the results of [8], particularly Theorem 5.5. To see this we first need the following definitions.

Let $1<p<\infty, 1<q<\infty ; 1 / p+1 / q \geqq 1$ and $1 / q+1 / q^{\prime}=1$.

We want to describe $\operatorname{Hom}_{L^{1}(\Gamma)}\left(L^{p}(\Gamma), L^{q^{\prime}}(\Gamma)\right)=M$. The ultraweak operator topology on $M$ is the topology generated by the seminorms of the form

$$
p(m)=\sum_{i=1}^{\infty}\left|\left\langle g_{i}, m f_{i}\right\rangle\right|
$$

where $g_{i} \in L^{q}(\Gamma), f_{i} \in L^{p}(\Gamma)$ and $\sum\left\|g_{i}\right\|\left\|f_{i}\right\|<\infty$. This topology corresponds to the weak* topology on $\left(\bar{L}^{q}(\Gamma) \otimes_{L^{1}(\Gamma)} L^{p}(\Gamma)\right)^{\prime}$ via the identification

$$
M \cong\left(\bar{L}^{q}(\Gamma) \otimes_{L^{1}(\Gamma)} L^{p}(\Gamma)\right)^{\prime} .
$$


Let $h \in \mathscr{K}(\Gamma)$ then the Riesz convexity theorem implies that for $f \in L^{p}(\Gamma)$, $f * h \in L^{q^{\prime}}(\Gamma)$ and

$$
\|f * h\|_{q^{\prime}} \leqq\left\|h^{\sim}\right\|_{q}^{p / q^{\prime}}\left\|\Delta^{-1 / p} h^{\sim}\right\|_{1}^{1-p / q^{\prime}}\|f\|_{p} .
$$

A locally compact group $\Gamma$ is said to satisfy property $\mathrm{P}_{p}^{q}$ (resp. $\mathrm{Q}_{p}^{q}$ ) if every element of $\operatorname{Hom}_{L^{1}(\Gamma)}\left(L^{p}(\Gamma), L^{q^{\prime}}(\Gamma)\right)$ can be approximated in the ultraweak (resp. weak) operator topology by operators of the form $f \mapsto f * h$ with $h \in \mathscr{K}(\Gamma)$.

We define a bilinear map $\theta: \bar{L}^{q}(\Gamma) \times L^{p}(\Gamma) \rightarrow L^{r}(\Gamma)$ where $1 / r=1 / p+1 / q-1$ by $\theta(g, f)=\Delta^{1 / p^{\prime}}\left(f^{\sim} * g\right)$ and then $\|\theta(g, f)\| \leqq\|f\|_{p}\|g\|_{q}$.

In conformity with the notation of Rieffel and others we let $A_{p}^{q}$ be the Banach space $H$ of Theorem 6 .

THEOREM 8. Let $\Gamma$ be a locally compact group. The following are equivalent conditions on $\Gamma$ :

(a) $\bar{L}^{q}(\Gamma) \tilde{\otimes}_{L^{1}(\Gamma)} L^{p}(\Gamma) \cong A_{p}^{q}$,

(b) $\Gamma$ satisfies property $\mathrm{P}_{p}^{q}$,

(c) $\Gamma$ satisfies property $\mathrm{Q}_{p}^{q}$.

Proof. We first show that (a) implies (b). Let $X$ be the subspace of

$$
\operatorname{Hom}_{L^{1}(\Gamma)}\left(L^{p}(\Gamma), \bar{L}^{q^{\prime}}(\Gamma)\right)
$$

of maps of the form $f \mapsto f * h$ with $h \in \mathscr{K}(\Gamma)$. If $\sum \Delta^{1 / p^{\prime}} f^{\sim} * g \in X^{0} \subset A_{p}^{q}$ then $\sum \Delta^{1 / p^{\prime}} f^{\sim} * g * h=0$ for all $h \in \mathscr{K}(\Gamma)$ and this means that $\sum \Delta^{1 / p^{\prime}} f^{\sim} * g=0$. By (b) of Theorem $6, \sum_{1}^{\infty}\left\langle g_{i}, m f_{i}\right\rangle=0$ for all $m \in \operatorname{Hom}_{L^{1}(\Gamma)}\left(L^{p}(\Gamma), \bar{L}^{q^{\prime}}(\Gamma)\right)$ and this says that $X^{00}=\operatorname{Hom}_{L^{1}(\Gamma)}\left(L^{p}(\Gamma), L^{q^{\prime}}(\Gamma)\right)\left(X^{0}\right.$ is the polar of $X$ in $\bar{L}^{q}(\Gamma) \tilde{\otimes}_{L^{1}(\Gamma)} \bar{L}^{p}(\Gamma)$, and $X^{00}$ its bipolar), which by the Bipolar Theorem is condition (b). Now (b) implies (c) trivially, and it is easy to see that (c) implies condition (c) of Theorem 6.

REMARK. As far as the author knows, it is unknown if every locally compact group satisfies $Q_{p}^{q}$. The most general known result is due to $C$. $S$. Herz who has shown that every amenable group has this property.

\section{REFERENCES}

1. N. Bourbaki, Livre V: Espace vectoriels topologique, Chaps. 1-5, Actualités Sci. Indust., no. 1189, Hermann, Paris, 1966. MR 34 \#3277.

2. - Livre VI: Intégration, Chaps. 1-8, Actualités Sci. Indust., nos. 1175, 1244, 1306, Hermann, Paris, 1965, 1967, 1963. MR 36 \#2763; MR 35 \#322; MR 31 \#3539.

3. B. Brainerd and R. E. Edwards, Linear operators which commute with translations. I: Representation theorems, J. Austral. Math. Soc. 6 (1966), 289-327. MR 34 \#6542.

4. G. Gaudry, Quasimeasures and operators commuting with convolution, Pacific J. Math. 18 (1966), 461-476. MR 34 \#3352.

5. R. Larsen, The multiplier problem, Lecture Notes in Math., No. 105, Springer-Verlag, Berlin, 1969.

6. L. Máté, The Arens product and multiplier operators, Studia Math. 28 (1966/67), 227-234. MR 35 \#5938. 
7. M. A. Rieffel, Induced Banach representations of Banach algebras and locally compact groups, J. Functional Analysis 1 (1967), 443-491. MR 36 \#6544.

8. - Multipliers and tensor products of $L^{p}$-spaces of locally compact groups, Studia Math. 33 (1969), 71-82. MR 39 \#6078.

9. R. Rigelhof, Induced representations of locally compact groups, Acta Math. 125 (1970), 155-188.

10. W. Rudin, Fourier analysis on groups, Interscience Tracts in Pure and Appl. Math., no. 12, Interscience, New York, 1962. MR 27 \#2808.

11. H. H. Schaefer, Topological vector spaces, Macmillan, New York, 1966. MR 33 \#1689.

Department of Mathematics, McGill University, Montreal, Quebec, Canada 